\title{
Intonational phonology in Bengali and English infant-directed speech
}

\author{
Kristine M. Yu ${ }^{1}$, Sameer ud Dowla Khan ${ }^{2}$, Megha Sundara ${ }^{3}$ \\ ${ }^{1}$ Department of Linguistics, University of Massachusetts, Amherst, MA, USA \\ ${ }^{2}$ Department of Linguistics, Reed College, Portland, OR, USA \\ ${ }^{3}$ Department of Linguistics, University of California, Los Angeles, CA, USA \\ krisyu@linguist.umass.edu, skhan@reed.edu, megha.sundara@humnet.ucla.edu
}

\begin{abstract}
We examined the phonetics and phonology of intonation of infant-directed speech (IDS) and non-IDS in story-reading in two typologically-divergent languages, English and Bengali. In addition to finding an increase in $\mathrm{f0}$ range and variability in IDS, replicating previous work on IDS prosody, we found novel evidence that $\mathrm{f0}$ manipulations in IDS are constrained by intonational phonology. Speakers in both languages used an increased proportion of tonal elements with higher tonal targets and more turning points in IDS, within the languagespecific intonational grammar. The tonal elements showing increased use in IDS also were associated with marking topic and focus. Thus, phonetic changes in IDS may in part be induced by speakers' choices of phonological tonal elements, which in turn may be connected with choices about marking discourse structure.
\end{abstract}

Index Terms: infant-directed speech, intonational phonology, speech style, information structure, Bengali, English

\section{Introduction and background}

It has long been noted that gradient phonetic variation in prosodic elements such as pitch, rhythm, and duration can carry information about interpersonal interaction and emotional state, cf. [1] for a review. However, work in intonational phonology ([1], [2], i.a.) has shown that prosodic variation is also structured according to the language-specific phonological grammar, which generates well-formed tunes constructed over discrete phonological categories, conveying linguistic meanings such as contrastive focus ([3], i.a.).

A classic example of gradient prosodic manipulation occurs in the speech style of infant-directed speech (IDS) or "motherese": relative to adult-directed speech, IDS has been shown to exhibit higher mean fundamental frequency (f0), higher maximum $\mathrm{f0}$, an expanded pitch range, greater f0 variability, and shorter utterances across a variety of languages ([4], [5], [6], i.a.). With almost no exception, work on IDS prosody has exclusively involved phonetic description of pitch manipulations as described above. Moreover, these gradient phonetic manipulations have been described as serving attentional and emotional functions. For instance, high pitch and increased pitch variability such as bell- and sinusoidalshaped pitch modulations have been suggested to maintain infant attention and elicit positive emotional rapport ([7], [8], [9], [10], [11], [12], [13]).

Without attention to intonational phonology, phonetic characterizations of IDS prosody leave open the possibility of pitch variation in IDS wholly motivated by attentional and emotional considerations, e.g. with sinusoidal ups and downs over a wide pitch range, irrespective of constraints of phonological grammar. To date, very little in the literature suggests otherwise, with the exception of three studies that explored phonological constraints on pitch variation in IDS. In the tone languages Thai [6] and Mandarin [14], it has been found that f0 cues to lexical tonal identity are preserved in IDS. Only one study thus far ([15]) addresses intonational phonological constraints on IDS; pitch range expansion in Japanese was shown to be restricted to boundary tones, a subcomponent of the intonational grammar where pitch is not lexically contrastive. In all three cases, the pitch manipulations ascribed to IDS did not interfere with lexically-contrastive aspects of pitch; the current study extends this recent work to two languages that have no lexical contrasts in pitch but that nevertheless manipulate pitch linguistically as intonation.

Our study investigates the hypothesis that pitch variability in IDS can be constrained by intonational phonological grammar in English and Bengali, languages with typologically divergent prosodic systems. We compared how parents of infants and young children read a story standardized across the languages in adult-directed speech (ADS) vs. IDS. We annotated the readings with intonational transcriptions based on intonational models of lab speech-style ADS in the two languages.

While [15] examined how pitch variation changes in Japanese IDS are conditioned on particular tonal elements, our study examines how pitch variation changes in IDS are realized via changes in the choice of tonal elements within intonational grammar. Moreover, our phonological analysis of IDS intonation allows us to examine how prosodic choices in IDS may reflect attention to conveying linguistic meaning and structure, in addition to regulating attention and emotional rapport. To preview our results: based on comparing the distribution of discrete tonal elements within the two speech styles, we found that in both languages, IDS showed an increase in tonal elements with high tonal targets and multiple turning points. These distributional changes occurred within the constraints of each language's intonational grammar. Moreover, these changes also resulted in an increase in the use of tonal elements used to mark aspects of discourse structure.

\subsection{English intonation and transcription}

The most widely known model of the intonation of American English (henceforth, simply "English") is often referred to as MAE_ToBI, from the Mainstream American English Tones and Break Indices transcription system devised to annotate the intonation of this variety ([1], [2], [3]). In MAE_ToBI, pitch accents are borne by the primary stressed syllable of prominent words, where stress is contrastive and thus must encode lexical information. The pitch accent inventory includes default $\mathrm{H}^{*}$ (high) and $\mathrm{L}^{*}$ (low) tones as well as rising $\mathrm{L}+\mathrm{H}^{*}$ (early rise) and $\mathrm{L}^{*}+\mathrm{H}$ (late rise) tones and one falling $\mathrm{H}+\mathrm{H}^{*}$ tone. The choice is related to attitude, focus status, and tonal environment ([3], [16], [17]). The choice of pitch accent is somewhat variable, although certain tune-meaning relationships have been proposed in the literature: $\mathrm{L}+\mathrm{H}^{*}$ and 
$\mathrm{L}^{*+\mathrm{H}}$ often convey focus ([3]). Boundary tones occur at the right edge of Intonation Phrases (IPs) and intermediate phrases (ip). The IP boundary tone inventory includes L-L\% (low falling), $\mathrm{H}-\mathrm{H} \%$ (high rising), $\mathrm{L}-\mathrm{H} \%$ (low rising), $\mathrm{H}-\mathrm{L} \%$ (high level), and !H-L\% (downstepped high level), the choice of which conveys sentence type, finality, etc. ([3]).

\subsection{Bengali intonation and transcription}

The intonational phonological model of Bangladeshi Standard Bengali (henceforth, simply "Bengali") presented here is based on that of [18], [19]. The pitch accent inventory of Bengali includes default $\mathrm{L}^{*}$ (low), $\mathrm{H}^{*}$ (high), $\mathrm{L}^{*}+\mathrm{H}$ (rising), as well as f-marked (focus-marking) versions of the latter two, $\mathrm{fH}^{*}$ (extra high) and $\mathrm{L}^{*}+\mathrm{fH}$ (rise to extra high), the choice of which conveys speaker attitude, focus status/type, and tonal environment ([18], [19]). Focus-marking tones are easily distinguished by their unique tonal interactions not seen in other pitch accents (i.e. violation of downtrend, immunity from overriding, triggering of post-focal tone compression); these phonetic and phonological criteria were used in the current study to identify focus-marking tones.

Each pitch accent projects a small prosodic unit called the Accentual Phrase (AP), which is marked on the right edge by a tone of the opposite target of the pitch accent: Ha (high target) and La (low target). These phrases group into ips, marked by sharp final contours: H- (sharp final rise) and L- (sharp final fall). As in English, ips group into IPs, marked by boundary tones that covey sentence type, information structure, and finality ([18], [19]). $\mathrm{H} \%$ (high rising) and $\mathrm{HL} \%$ (high falling) can serve as topicalizers, and both $\mathrm{LH} \%$ (low rising) and $\mathrm{HLH} \%$ (high dipping) can serve as markers of non-finality.

\subsection{Comparison of English and Bengali intonation}

Noteworthy similarities and differences between Bengali and English intonation are in (a) pitch contour regularity, (b) focus realization, and (c) boundary tone complexity.

One of the most clearly noticeable differences between English and Bengali is the regular repeating patterns seen in the Bengali pitch contour and the general lack of such regularity in English. This is largely an effect of the restricted pitch accent and AP boundary tone distribution of Bengali. While a content word in English is fairly free to bear any of five basic pitch accents $\left(\mathrm{H}^{*}, \mathrm{~L}^{*}, \mathrm{~L}+\mathrm{H}^{*}, \mathrm{~L}^{*}+\mathrm{H}, \mathrm{H}+\mathrm{H}^{*}\right)$ or none, Bengali content words almost always bear $\mathrm{L}^{*}$ followed by a $\mathrm{Ha}$, except in very specific cases: $\mathrm{H}^{*}$ followed by $\mathrm{La}$ conveys surprise or sarcasm, and $\mathrm{L}^{*+\mathrm{H}}$ is only a secondary option phrase-finally, and the two f-marked pitch accents (fH*, $\mathrm{L}^{*}+\mathrm{fH}$ ) are only used to convey narrow focus.

In both languages, focus is realized via pitch accent choice and post-focal tone compression (i.e. deaccenting). In Bengali, an $\mathrm{f}$-marked tone $\left(\mathrm{fH}^{*}, \mathrm{~L}^{*}+\mathrm{fH}\right)$ marks the focused word. These are sharply distinct from the non-focused pitch accent $L^{*}$, and can even be distinguished from $\mathrm{H}^{*}$ and $\mathrm{L}^{*}+\mathrm{H}$ as the latter two obey downtrend and do not trigger post-focal compression, unlike f-marked tones. In English, the focused element can bear $\mathrm{L}+\mathrm{H}^{*}$ and $\mathrm{L}^{*}+\mathrm{H}$ rather than $\mathrm{H}^{*}$ in declaratives [3]. The distinction between tones associated with and without focus in Bengali is clearer than in English, where $\mathrm{H}^{*}$ and $\mathrm{L}+\mathrm{H}^{*}$ appear to have overlapping allophonic variation [20], although they are functionally distinct [21].

Lastly, Bengali and English differ in the complexity of their boundary tones. While Bengali has three levels of tonally-marked prosodic structure (i.e. AP, ip, IP), English has only two (i.e. ip, IP). Bengali APs and ips typically end in a single underlying tone, but the ip tones $\mathrm{L}-$ and $\mathrm{H}$ - both inherently incorporate a sharp phrase-final rise or fall in pitch. English ip tones L- and H- do not have this sharp phrase-final rise or fall, and are instead realized as "phrase accents", i.e. generally low or generally high pitch across the phrase-final stretch. The five Bengali IP tones are very complex, including one, two, or three tonal targets (e.g. HLH\%), all of them involving large changes in pitch. The five English IP tones, on the other hand, can have only one or two tonal targets (e.g. L$\mathrm{H} \%$ ), and two of them involve a stretch of flat pitch (H-L\%, !H-L\%), which is at best rare in Bengali.

\section{Methods}

\subsection{Data collection}

The speech of nine (5 male, 5 female) native speakers of English and ten (5 male, 5 female) native speakers of Bengali was recorded in a quiet room in Los Angeles. Subjects were paid $\$ 5$ for their participation, which typically took $20 \mathrm{~min}$. To help ensure that subjects were comfortable and familiar with the IDS task, only parents were recruited. The English speakers had infants of $4.4 \pm 0.7$ months of age at the time of recording, while there was no specific cutoff for the age of the Bengali speakers' children due to subject pool limitations. However, all Bengali subjects lived with their (grand)children $<10$ yrs of age, and six subjects were teachers at a Bengalilanguage weekend school for children.

Subjects were recorded while reading the "North Wind and Sun" fable, the Bengali version of which was taken from [22]. Storytelling was used as a context appropriate for both IDS and non-IDS default read speech. The particular text was chosen for comparison with other work on speech rhythm using the same text, as part of a larger study. Using translations of the same text across both languages ensured that the recordings from the two languages would not be affected by different semantic/pragmatic features triggered by reading different stories. Furthermore, unlike studies that examine spontaneous IDS, the current study kept the text constant to minimize changes in semantics, morphosyntax, and the underlying segments across conditions. This way we could observe how speakers' prosodic choices might change, given the same morphosyntactic and discourse structure.

Multiple recordings were made using a Shure SM10A head-mounted microphone plugged into a laptop computer via a preamplifier. The first of the two conditions was default reading ("non-IDS"), in which subjects were asked to "read at a comfortable pace". This task direction was designed to be comparable with other studies of speech rhythm using lab speech, as part of a larger study. The second condition was simulated infant-directed reading ("IDS"), in which the subject was asked to read the same passage as if speaking to their 4-5 month-old infant. (Bengali speakers were asked to imagine their child at that age.). This is an age range where IDS has fewer single-word utterances, is less dominated by soothing/comforting affect, and is intensely used for rapport and attention ([9], [6]). Thus, it is an age range where paralinguistic demands play a large role in driving prosodic 
manipulations in IDS. Childlike illustrations and plush toys were used to help further encourage this register. ${ }^{1}$

\subsection{Data analysis}

For each condition, the three clearest repetitions with minimal or no disfluencies were analyzed, giving 2 conditions $\mathrm{x} 3$ repetitions $=6$ recordings per speaker. Text grids were generated in Praat ([23]) to allow annotation in MAE_ToBI tone labels ([24]) for English and B-ToBI tone labels ([18], [19]) for Bengali, by a transcriber trained in each system.

Both acoustic-phonetic measurements and categorical phonological measurements were taken during each sound file. These include: pitch range, tonal inventory, total number of pitch accents and boundary tones, and the frequency of use of each type of pitch accent and boundary tone. Currently, the results presented reflect the whole Bengali data set, but annotations for the English data set are incomplete and preliminary results presented here reflect one repetition from each speech style for each speaker. For the Bengali data, a random sample of the data has also been transcribed by a second transcriber to check for intertranscriber reliability.

Transcribers in both languages were trained only on nonIDS speech materials from outside of the current study, and were encouraged to propose new tones or take note of new tonal interactions when approaching the IDS and non-IDS recordings from the current study. This was done to discourage transcribers from forcing aspects of the non-IDS intonational phonological grammar onto what could hypothetically be a completely different grammar in IDS. All tones transcribed in IDS were also found in the non-IDS recordings in this study, including two tones in Bengali that had not yet been identified in previous work (and were not found in the training files): $\mathrm{L}+\mathrm{H}^{*}, \mathrm{M} \%$.

To analyze changes between the distribution of pitch accent and boundary tone types between styles, proportions of each tone type were calculated, aggregated across repetitions. Separate mixed effects logistic models were built for individual tonal elements, with the proportion of an individual tonal element as the dependent variable, speech style and speaker sex as fixed effects, and random effects by speaker (cf. [25] for advantages of logistic models for proportional data). Results reported as significant were significant with Bonferroni corrections for multiple comparisons.

\section{Results}

\subsection{Phonetic changes in IDS: both languages}

F0 data was extracted at $10 \mathrm{~ms}$ intervals from the recorded files using RAPT ([26]). Mean, minimum, and maximum f0 (in $\mathrm{Hz}$ ), as well as $\mathrm{f0}$ range and change in f0 variability were calculated over each phrase and averaged across phrases for each speaker, following [5]. Paired t-tests indicated significant increases in IDS for mean $\mathrm{f} 0$, maximum $\mathrm{f0}$, f0 range, and f0 variability, all with $\mathrm{p}<0.01$, but no change in minimum fo, for

${ }^{1}$ In pilot work when an infant was in the room, the infant would often fuss and cry, disturbing the audio recording, causing disfluencies in the subject's speech, and interrupting $\mathrm{him} / \mathrm{her}$ at unpredictable intervals. As the study of intonational phonology in IDS is in its beginning stages, we decided to abstract away from these disturbances for the time being. both languages. These results are consistent with previous studies of IDS in many languages, supporting the validity of properties of the simulated IDS recorded here as bearing on properties of IDS in general.

\subsection{Phonological changes in IDS: Bengali}

While the number of pitch accents significantly decreased by $7 \%$ in IDS $(t(9)=3.1, p=0.01)$, the proportion of focus-marking pitch accents significantly increased. Proportions of pitch accents between speech styles are shown below in Figure 1. The proportion of $\mathrm{fH}^{*}$ accents significantly increased from an average of $0.9 \%$ across speakers to $5.0 \%$ in IDS $\left(\beta_{\text {IDS }}=2.1, p\right.$ $=2 \mathrm{e}-6)$. The proportion of $\mathrm{L}^{*}+\mathrm{fH}$ accents significantly increased from $1.9 \%$ to $4.1 \%$, with a larger increase for females $\left(\beta_{\text {IDS nested in SEX }}=1.0, p=0.007\right)$. The proportion of (default) L* accents significantly decreased, while the proportion of (non-default) $\mathrm{H}^{*}$ accents significantly increased.

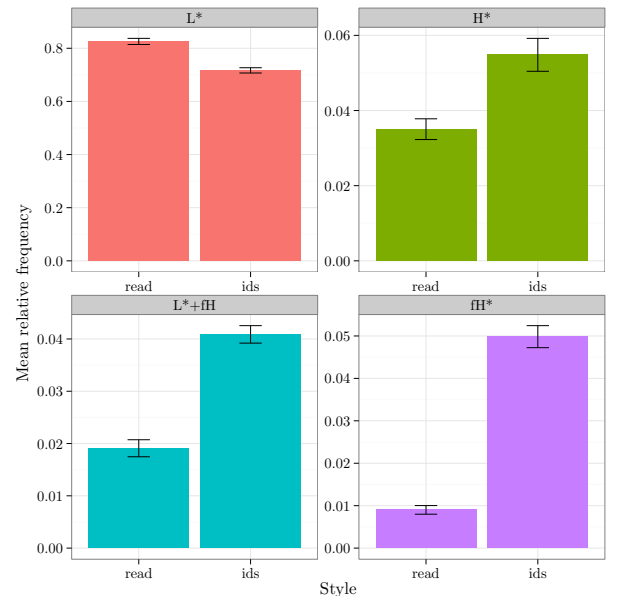

Fig. 1: Change in relative frequency of pitch accents between speech styles, averaged over speakers. Error bars show $\pm 1 \mathrm{SE}$.

In addition, the number of IPs significantly increased by $49 \%$ in IDS $(\mathrm{t}(9)=5.5, \mathrm{p}=3.8 \mathrm{e}-4)$ and the number of ips by $21 \%$ $(t(9)=-3.5, p=0.006)$, while the number of APs significantly decreased by $10 \%$ in IDS $(\mathrm{t}(9)=-3.5, \mathrm{p}=6.5 \mathrm{e}-3)$. Among the AP tone types, there was no significant change in the proportion of $\mathrm{La}$ tones, but the proportion of $\mathrm{Ha}$ tones significantly decreased in IDS ( $\left.\beta_{\text {IDS }}=-0.64, p=7 e-8\right)$, while the proportion of $\mathrm{fHa}$ tones significantly increased $\left(\beta_{\mathrm{IDS}}=\right.$ $0.79, p=2 \mathrm{e}-8)$. Changes in the proportion of IP tone types are shown in Figure 2. The proportion of $\mathrm{HLH} \%$ tones significantly increased from an average of $8 \%$ to $17 \%$ in IDS $\left(\beta_{\mathrm{IDS}}=0.80, \mathrm{p}=1.9 \mathrm{e}-5\right)$, as did the proportion of HL\% tones, from an average of $2 \%$ to $7 \%\left(\beta_{\mathrm{IDS}}=1.2, \mathrm{p}=3.1 \mathrm{e}-4\right)$. In contrast, the proportion of $\mathrm{L} \%$ and $\mathrm{LH} \%$ tones significantly decreased $\left(\beta_{\text {IDS }}=-0.31, p=7.2 \mathrm{e}-3\right.$. $\left.\beta_{\text {IDS }}=-0.58, p=3.9 e-6\right)$.

\subsection{Phonological changes in IDS: English}

While the number of pitch accents did not significantly change between speech styles, $(\mathrm{t}(8)=-1.8, \mathrm{p}=0.11)$, there was a significant increase in IDS in the proportion of rising pitch accents of $\mathrm{L}^{*}+\mathrm{H}$ from $0.7 \%$ to $4.9 \%$ on average across speakers, and of $\mathrm{L}+\mathrm{H}^{*}$ from $2.8 \%$ to $10.3 \%$ on average $\left(\mathrm{L}^{*}+\mathrm{H}: \beta_{\mathrm{IDS}}=1.9, \mathrm{p}=0.003 ; \mathrm{L}+\mathrm{H}^{*}: \beta_{\mathrm{IDS}}=1.44, \mathrm{p}=4.17 \mathrm{e}-5\right)$. There was a concomitant insignificant trend for decreases in IDS in the proportion of $\mathrm{L}^{*}$ and $\mathrm{H}^{*}$ accents. In addition, speakers produced a significantly higher average of $32 \%$ more 
ips and 35\% more IPs in IDS (ip: $\mathrm{t}(8)=3.4, \mathrm{p}=0.01$, IP: $\mathrm{t}(8)$ $=4.8, \mathrm{p}=0.001)$. There were no significant changes in the proportion of different boundary tone sequences, and the majority of boundary tone sequences were L-L\% (85\%).

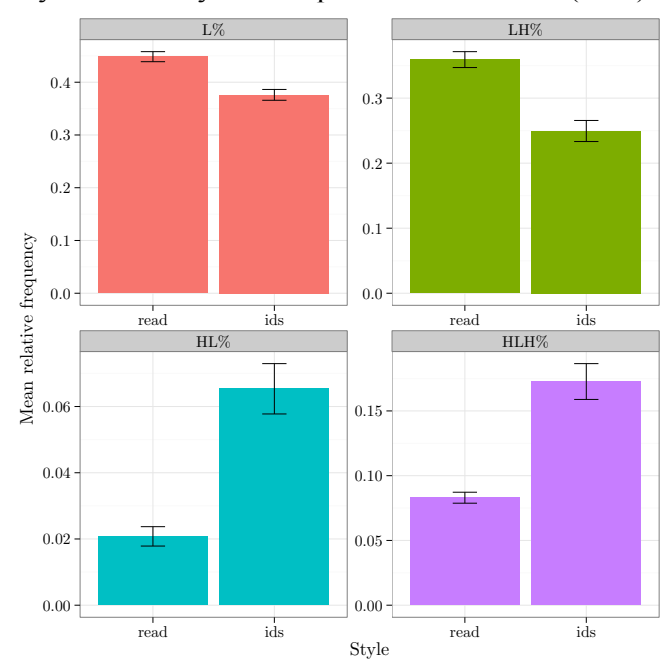

Figure 2: Change in relative frequency of IP tones between speech styles averaged over speakers. Error bars show $\pm 1 \mathrm{SE}$.

\subsection{Summary of results}

To summarize, both languages showed an overall acousticphonetic expansion of the pitch range and overall increase in f0 variability. Both languages also had phonological changes in intonation: increases in the use of non-default pitch accents (despite the lack of increase in pitch accents overall), increases in the number of ips and IPs, and for Bengali, increases in the use of some complex boundary tones.

\section{Discussion}

We found that IDS prosody in a storytelling context was characterized by gradient phonetic changes as well as changes in the choice of discrete tonal elements within the languagespecific intonational grammar.

The overall expansion of f0 range, higher f0 maxima and increased fo variability in IDS was realized within the constraints of the language-specific grammar. First, we found that pitch variation in IDS could be understood using phonological models of intonation: all tones we found in IDS were also found in non-IDS. Second, changes in the choice of tonal elements in IDS were changes that could induce the gradient phonetic changes of higher f0 maxima and increased fo variability. Both languages showed an increase in pitch accents with higher tonal targets and more turning points in IDS $\left(\mathrm{L}+\mathrm{H}^{*}\right.$ and $\mathrm{L}^{*}+\mathrm{H}$ in English; $\mathrm{H}^{*}, \mathrm{fH}^{*}$ and $\mathrm{L}^{*}+\mathrm{fH}$ in Bengali); Bengali also had an increase in use of an AP tone with a higher tonal target, fHa. In addition, both languages showed increases in the number of higher-level boundary tones. Higher-level boundary tones such as IP tones are realized in an expanded pitch range relative to lower-level boundary tones. Moreover, in Bengali, the inventory of IP tones includes complex contours not available for lower-level boundary tones; indeed, there was a decrease in default $\mathrm{L} \%$ tones but an increase in $\mathrm{HL} \%$ and $\mathrm{HLH} \%$ tones in IDS. Thus, while non-IDS in Bengali was characterized by a series of predictable contours built with $\mathrm{L}^{*}, \mathrm{Ha}$, and $\mathrm{L} \%$, IDS replaced these with $\mathrm{H}^{*} / \mathrm{fH}^{*}$ and complex boundary tones.
In addition, the change in choice of tonal elements in IDS appears to not only be influenced by the pitch patterns they induce, but also by their tone-meaning mappings. Almost all the pitch accents with increased use in IDS in both languages are ones associated with focus, and Bengali also showed an increase in use of the focus-marking fHa AP tone and the topicalization marker HL\%. The lack of an increase in the number of pitch accents in both languages can also be understood as a consequence of focus-marking within intonational grammar: preliminary analyses suggest that the increased use of focus-marking pitch accents in both English and Bengali in IDS limited the increase in the number of pitch accents overall since the use of focus-marking pitch accents causes post-focal tonal compression and deaccenting in both Bengali and English. Preliminary inspection also suggests that speakers were not deploying tones associated with focus- and topic-marking in IDS in a manner divorced from the discourse structure, which is what one might expect if these tones were chosen simply due to the pitch patterns they induce. For instance, $\mathrm{L}+\mathrm{H}^{*}$ accents in English did not appear on pronouns, which were discourse-given. Thus, we speculate that the phonetic pitch changes in IDS may be in part a consequence of increased marking of discourse structure in IDS, as suggested in [10].

\section{Conclusions}

In this study, we examined changes in patterns of pitch variation in English and Bengali when speakers read a story in a non-infant directed style and an infant-directed style. We found that in both languages, there was an increase in f0 range via raising of f0 maxima as well as increased f0 variability in IDS, replicating previous cross-linguistic phonetic work on IDS prosody. In addition, we performed phonological analyses of intonation in the two speech styles, which revealed that the phonetic pitch manipulations in IDS we observed were constrained by language-specific intonational grammar. We observed that IDS and non-IDS intonation could be modeled with the same underlying tonal elements, but that the distribution of use of these elements differed between styles. In IDS, speakers increased the proportion of tonal elements with high tonal targets and more turning points, which induce higher f0 maxima and greater f0 variability. Thus, the phonetic changes in pitch in IDS may have been in part a consequence of speakers' choice of phonological tonal elements. Moreover, tonal elements with increased use in IDS were also associated with marking focus and topic in previous work. Preliminary analyses suggest that these tonal elements were not placed arbitrarily, but in ways consistent with them being used to mark discourse structure. Thus, phonetic changes in pitch in storytelling IDS may also be in part due to increased marking of discourse structure. In further work, we are continuing to analyze the role of discourse and syntactic structure in the prosodic choices made by the speakers, and we are performing phonetic analyses of $\mathrm{f} 0$ assessing the contribution of different tonal elements to increasing f0 range and variability.

\section{Acknowledgements}

Special thanks to Alejna Brugos, J'aime Panna Roemer and Megan Keough for help in recording and annotating the sound files. 


\section{References}

[1] Ladd, D. Robert. Intonational phonology, Cambridge: Cambridge University Press, 1996.

[2] Pierrehumbert, J.B. "The phonology and phonetics of English intonation", Ph.D. dissertation, Massachusetts Institute of Technology, Cambridge, MA, 1980.

[3] Pierrehumbert, J. and Hirschberg, J. "The meaning of intonational contours in the interpretation of discourse", in P. Cohen, J. Morgan, and M. Pollack [Ed], Intentions in Communication, 271-311, 1990

[4] Fernald, A. and Simon, T. "Expanded intonation contours in mothers' speech to newborns,” Dev. Psych., 20(1): 104-113, 1984.

[5] Fernald, A., Taeschner, T., Dunn, J., Papousek, M., BoyssonBardies, B., and Fukui, I. "A cross-language study of prosodic modifications in mothers' and fathers' speech to preverbal infants", J. Child Lang., 16: 477-501, 1989.

[6] Kitamura, C., Thanavishuth, C., Burnham, D. and Luksaneeyanawin, S. "Universality and specificity in infantdirected speech: Pitch modifications as a function of infant age and sex in a tonal and non-tonal language", Inf. Behav. and Dev., 24(4): 372-392, 2002.

[7] Fernald, A. \& Kuhl, P. "Acoustic determinants of infant preference for motherese speech", Inf. Behav. and Dev., 10: 279293, 1987.

[8] Stern, D.N., Spieker, S., Barnett, R. K., and MacKain, K. "The prosody of maternal speech: infant age and context related changes," J. Child Lang., 10: 1-15, 1983.

[9] Stern, D.N., Spieker S., and MacKain, K. "Intonation contours as signals in maternal speech to prelinguistic infants", Dev. Psych., 18(5): 727-735, 1982.

[10] Fernald A., and Mazzie, C. "Prosody and focus in speech to infants and adults", Dev. Psych., 27(2): 209-221, 1991.

[11] Werker, J.F. and McLeod, P.J. "Infant preference for both male and female infant-directed talk: a developmental study of attentional and affective responsiveness", Can. J. Psychol., 43(2): 230-246, 1989.

[12] Fernald, A. "Intonation and communicative intent in mothers' speech to infants: is the melody the message?", Child Dev., 60: 1497-1510, 1989

[13] Trainor, L.J., Austin, C.M., and Desjardins, R.N. "Is infantdirected speech prosody a result of the vocal expression of emotion", Psych. Sci., 11(3): 188-195, 2000.

[14] Liu, H., Tsao, F., and Kuhl, P.K. "Acoustic analysis of lexical tone in Mandarin infant-directed speech", Dev. Psych., 43(4): 912-917, 2007.

[15] Igarashi, Y., Nishikawa K., Tanaka K., and Mazuka R., "Phonological theory informs the analysis of intonational exaggeration in Japanese infant-directed speech", J. of the Acoustical Soc. of America, 134(2): 1283-1294, 2013.

[16] Dainora, A. "Does intonational meaning come from tones or tunes? Evidence against a compositional approach", Speech Prosody 2002, 2002

[17] Dainora, A. "Modeling intonation in English: a probabilistic approach to phonological competence", in Goldstein, L., Whalen, D.H., Best, Catherine T. [Ed], Laboratory Phonology 8, Mouton de Gruyter, 2006.

[18] Khan, S.D. "Intonational phonology and focus prosody of Bengali", Ph.D. dissertation, University of California, Los Angeles, CA, 2008

[19] Khan, S.D. "The intonational phonology of Bangladeshi Standard Bengali", in Jun, S.-A. [Ed], Prosodic Typology II: The Phonology of Intonation and Phrasing, Oxford Univ. Press, 2014 in press.

[20] Bartels, C. and Kingston, J. "Salient pitch cues in the perception of contrastive focus," in Bosch, P. and van der Sandt, R. [Ed], Focus and natural language processing, IBM Deutschland. 1994

[21] Ito, K., Bibyk, S.A., Wagner, L., and Speer, S.S. "Interpretation of contrastive pitch accent in six to eleven-year-old English- speaking children (and adults)", J. Child Lang., 41(1): 84-110, 2014

[22] Khan, S.D. "Bengali (Bangladeshi Standard)", JIPA, 40(2), 221$225,2010$.

[23] Boersma, P. "Praat, a system for doing phonetics by computer", Glot International, 5(9/10): 341-345, 2001.

[24] Beckman, M. and Ayers, G. Guidelines for ToBI labeling, 1997, http://www.ling.ohiostate.edu// tobi/ame_tobi/labelling_guide_v3.pdf.

[25] Jaeger, F. "Categorical Data Analysis: Away from ANOVAs (transformation or not) and towards Logit Mixed Models", J. Mem. \& Lang., 59(4): 434-446.

[26] Talkin, D. "A robust algorithm for pitch tracking (RAPT)", in Kleijn, W. B. and Paliwal, K. K. [Ed], Speech coding and synthesis, Elsevier, 1995. 